\title{
Developmental Lumbar Spinal Canal Stenosis and Severity of Radiculopathy
}

\section{Muthuuri Jamlick}

The Mombasa Hospital

Correspondence to: Dr. Jamlick Muthuuri, The Mombasa Hospital, PO Box 84074-80100, Mombasa, Kenya; email: michenimuthuuri@yahoo.com

\begin{abstract}
Background: The severity of radiculopathy is varied and may not concur with MRI findings. Large disc bulges may elicit fewer symptoms than smaller bulges. This was postulated to be due to the size of the canal. Objective: To assess the severity of radiculopathy in individuals with developmental lumbar spinal canal stenosis (DLSS) and in those without. Methods: This cross-sectional survey compared two groups: those with DLSS and those without. 597 participants were recruited and screened for DLSS, creating two groups: a group with DLSS and a group without. Severity and duration of symptoms were assessed with visual analogue scale, Oswestry Disability Index and duration of symptoms. Results: Prevalence of DLSS in the study population was $19 \% .90 \%$ of those with DLSS had radiculopathy with longer duration of symptoms (mean
\end{abstract}

\section{Introduction}

Lumbar spinal stenosis is defined as a narrowing of the spinal canal that results in compression of the neurovascular tissues before their exit or as they exit the spinal canal. Arnoldi et al. presented an etiological classification that attempted to distinguish congenital, developmental and acquired forms of spinal stenosis $(1,2)$. Congenitally undeveloped vertebral canals tend to be syndromic, particularly associated with dwarfism and achondroplasia (3). Developmentally narrow canals are characterized by narrowed spinal canals when compared with fully developed canals in the same population. The cause is unknown but it is thought to be part of generalized osseous stunting. Because the depth of the canal or the anteroposterior diameter (AP diameter) is fully completed by 5 years of age and has no chance of catching up, it is vulnerable to early life stresses such as malnutrition (4). Malnutrition may be caused by food shortage, placenta disorders and various maternal factors; these all may potentially influence development of the spinal canal (5). The width of the spinal canal diameter (transverse diameter) continues to grow until around 17 years (6), giving it room to grow when nutritional circumstances change. In developmentally narrow canals, the depth is more affected, and therefore statistical developmental lumbar spinal canal stenosis (DLSS) is narrowness of -2 SD from the population mean of depth of the vertebral canal. The resultant canal is either ovoid or shows trefoilness. In this study, the prevalence of DLSS (in the study population) was found to be $19 \%$. Lumbar spinal canal stenosis is an important etiological factor
$20.8 \pm 21.8$ months against $12.3 \pm 15.9$ months, $\mathrm{p}<0.0001$ ), higher ODI scores (32.9 \pm 15.2 against $17.2 \pm 16.5, \mathrm{p}<0.0001)$, and a higher VAS score (5.1 \pm 2.9 vs $4.7 \pm 1.3, p=0.068)$. Conclusions: Patients with DLSS have increased prevalence of radiculopathy and symptoms are more severe than in those without.

Key words: Developmental, Lumbar spinal canal, Radiculopathy, Chronicity, Disability, Severity

Ann Afr Surg. 2019; 16(2):64-68

DOI: http://dx.doi.org/10.4314/aas.v16i2.5

\section{Conflicts of Interest: None}

Funding: None

(C) 2019 Author. This work is licensed under the Creative Commons Attribution 4.0 International License

in chronic low back pain syndromes (3, 7-9). Using ultrasound scanning, Porter et al. examined thousands of patients attending low back pain clinic and found a significant difference in the canal size of symptomatic individuals than in asymptomatic volunteers. They also found that most patients requiring surgery have smaller spinal canal cross-sectional areas (10). It can be concluded that canal size is a crucial determinant of neurological outcome in symptomatic individuals.

Various methods have been used to measure the dimensions of the spinal canal including ultrasonography, plain X-ray, CT scan and MRI, but the radiological method of choice for evaluating spinal canal stenosis and its pathology is MRI (11). Advantages of MRI over other methods include: noninvasiveness, non-radiation, high sensitivity and high soft tissue contrast that clearly shows nervous tissue, ligaments and other paraspinal soft tissues $(11,12)$. Except for the CT scan radiating, which is a major disadvantage, $\mathrm{CT}$ axial views are an accurate method of measuring canal dimensions. In both CT and MRI scans, measurements are taken at the interpedicular level where the canal is spared the degenerative processes that generally affect the junctional levels, particularly the disc level (13). This study used CT and MRI scans.

The visual analogue scale (VAS) and the Oswestry Disability Index (ODI) have been validated to measure the health-related quality of life outcomes. VAS evaluates severity of pain while ODI evaluates a patient's functional disability. Duration of symptoms is an unspecific measure of chronicity of the disease condition. 


\section{Methods}

The study was a cross-sectional survey (all data collected in a single short period) and was part of a larger research study with multiple objectives.

Data were collected between 2 October 2017 and 13 January 2018 in seven radiological centres in the coastal region of Kenya. Permission was sought from the Ethics Review Committee (ERC) of Mount Kenya University and the study permit was granted by the National Commission for Science, Technology and Innovation. Written informed consent was sought from each participant, who joined the study freely without coercion or monetary inducement. Confidentiality was maintained and patient information de-identified. All study participants were mature adults who had a CT or MRI scan of the lumbosacral spine to allow measurement of the spinal canal. The study used a semi-structured questionnaire that was administered by a researcher to collect data. All necessary observations and measurements were collected simultaneously on the spot. The questionnaire had sections for VAS enquiry, longevity of symptoms, and an ODI questionnaire attached.

All participants were indigenous adult black Africans who were born in and and spent their first 5 years of life in the coast region (this was hoped to eliminate genetic confounders). The sample was meant to represent an impoverished population because poverty levels in the coastal region are higher than the national average for the country ( $46.5 \%$ vs. $42 \%)(24)$. The individuals were required to be between 18 and 60 years of age and have had a CT or MRI scan with clear magnification of the lumbosacral spine. Those excluded were people of non-African race, those with musculoskeletal disorders such as hip dysplasia or scoliosis, those who were syndromic (Downs syndrome, dwarfism and achondroplasia), and those with prior spine surgery, spine fractures, infection or tumors. Using Fisher's formula, the sample size was predetermined as 436. The recruitment obtained 597 participants and the study sample was obtained from this group by systematic sampling method, where every 5 th participant was eliminated beginning from a random number. Lumbar spinal canal dimensions were measured prospectively using a Siemens sixteen-slice multi-detector CT scanner or a GE 1.5T MRI scanner (General Electric, USA). Measurements of the spinal canal were taken at the interpedicular level on the axial scans of each lumbar vertebra from LV1 to LV5. All measurements were done manually by study assistants and counterchecked by the principal researcher. The true value of the measurement was recorded after factoring in the magnification.

The dimensions of the spinal canal were marked anteriorly by the posterior edge of the vertebral body, posteriorly by the anterior edge of the spinous process, and laterally by the medial borders of the pedicles. Measurements used those landmarks on the axial scans of each lumbar vertebra from LV1 to LV5 at the pedicular level (Fig. 1).

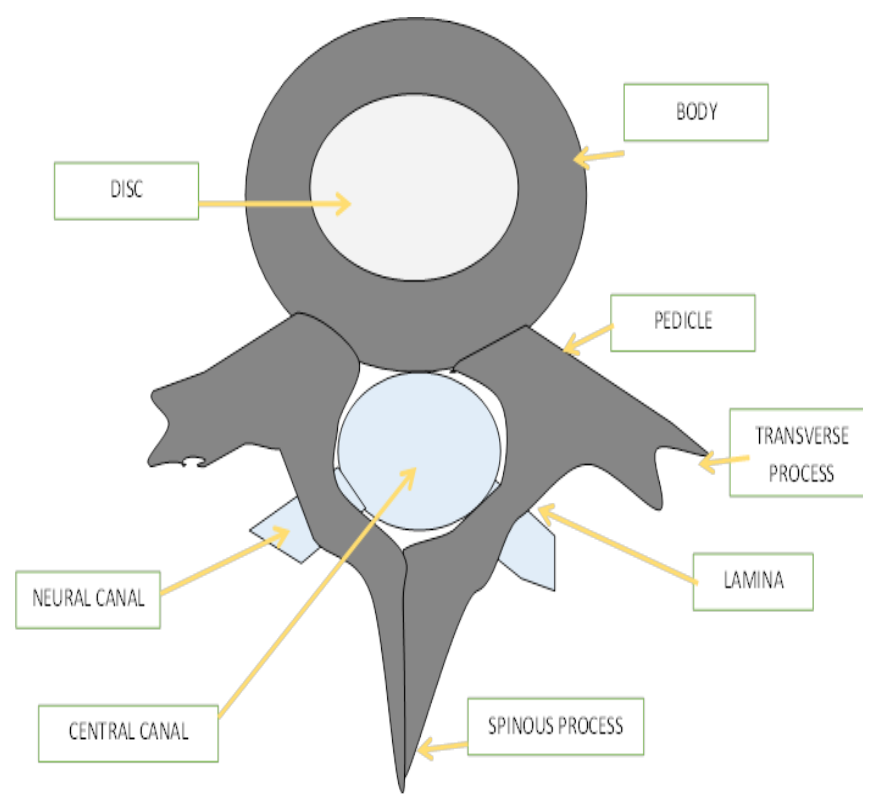

Figure 1. Anatomy of a typical lumbar spine. Note the central spinal canal and its boundaries.

The anteroposterior diameter (APD) and the transverse diameter (TRD) were taken for each segment of the lumbar spine in each participant. These dimensions were used to calculate the cross-sectional area (CSA) using the mathematical formula for calculating the area of an oblong as follows: $\mathrm{A}=\pi \mathrm{xy}$, where $\mathrm{A}$ is the cross-sectional area, $x$ the transverse diameter and $y$ the anteroposterior diameter (Fig. 2).

After the spinal canal measurements, two groups were created: Group1 consisted of individuals with DLSS and Group2 of individuals without DLSS. Comparisons were made within these groups to determine the severity of radiculopathy symptoms. Data were analyzed using IBM SPSS version 20.0 (SPSS Inc., Chicago, Illinois, US). Descriptive statistics

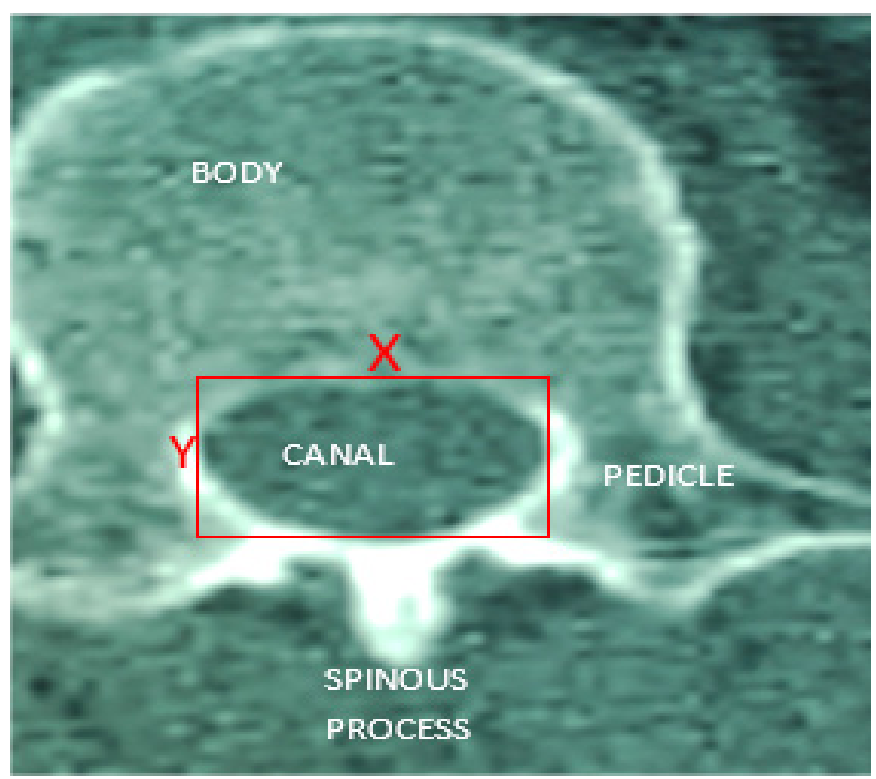

Figure 2. Spinal canal measurement in an axial CT scan of a lumbar vertebra at the interpedicular level. $\mathrm{Y}$ is the anteroposterior diameter while $X$ is the transverse diameter of the canal. The red box demonstrates the method of measurement and the parameters measured. 
were used to analyze for frequencies, central tendency (mean, median and mode), and dispersion (standard error, standard deviation, range and sample variance). Independent samples $t$-test was used to compare means for the two groups. $\mathrm{p}<0.05$ was considered statistically significant.

The procedures followed in this study were in accordance with the ethical standards of the responsible committee on human experimentation (institutional and national) and with the Helsinki Declaration of 1964, as revised in subsequent editions.

\section{Results}

The sample had 217 males and 219 females $(n=436)$ with a mean age of 43 years. Three hundred fifty-four (81\%) participants were symptomatic: 109 (23\%) had isolated low back pain; 193 (44\%) had back pain and radiculopathy; 52 participants (12\%) had isolated radiculopathy without back pain. Eighty-two participants (19\%) had no symptoms.

After screening (for DLSS), 84 participants (19\%) were below $-2 \mathrm{SD}$ of mean and formed Group1. The remaining 354 were statistically negative and formed Group 2.

The overall rate of radiculopathy in the study population was $56 \%$. The frequency of radiculopathy in Group 1 (DLSS) was compared with Group 2 (without DLSS). Ninety percent of individuals with DLSS had radiculopathy compared with $48 \%$ in those without DLSS. The differences were statistically significant with chi square in cross-tabulation returning a $\mathrm{p}$ $<0.0001$.

The mean APD in the normative population was $13.8 \pm 2.5 \mathrm{~mm}$ with a TRD of $17.8 \pm 3.6 \mathrm{~mm}$, giving mean CSA of $192 \pm 70 \mathrm{~mm}^{3}$. The mean APD in Group1 $(n=84)$ was $13.1 \pm 2.5 \mathrm{~mm}$ and $14.4 \pm 2.6 \mathrm{~mm}$ in Group2 $(n=352), \mathrm{p}=0.046$. The mean TRD in Group1 was $17.5 \pm 3.3 \mathrm{~mm}$ and in Group2 $18.0 \pm 3.8 \mathrm{~mm}$ $(\mathrm{p}=0.176)$. The mean CSA in Group1 was $181.5 \mathrm{~mm}^{2}$ and 203.8 $\mathrm{mm}^{2}$ in Group2 $(\mathrm{p}=0.042)$. In addition, those with isolated radiculopathy $(n=52)$ had a mean CSA of $174.8 \mathrm{~mm}^{2}$ and those without any symptoms $(n=82) 201.6 \mathrm{~mm}^{2}(\mathrm{p}<0.0001)$.

\section{Disease burden}

The disease burden to the individual was measured by determining severity of pain at presentation, chronicity and disability. Severity of pain was measured using VSA while chronicity was measured as duration of symptoms (in months) of low back pain and radiculopathy or radiculopathy alone. Disability was measured using the Oswestry disability index, which measures deterioration in activities of daily living.

\section{DLSS and chronic low back pain (LBP)}

Out of the 84 cases of DLSS, 69 (82\%) suffered LBP compared with $66 \%$ of those without DLSS. The difference was statistically significant $(\mathrm{p}=0.0044)$. Although there is a difference between the two groups, LBP appears to be a highly prevalent condition in the population ( $72 \%)$, where DLSS is a significant but not the only cause.
Radiculopathy (radiculopathy/myelopathy symptoms)

Out of the 84 cases of DLSS, 76 (90\%) suffered radiculopathy compared with $48 \%$ of those without DLSS. The difference was statistically significant with chi-square in cross-tabulation returning $\mathrm{p}<0.001$. As expected, DLSS is major contributor to the radiculopathy syndrome, but the $48 \%$ prevalence in the non-DLSS population implies that other causes of nervous tissue compression exist.

\section{Severity}

VSA showed that those with DLSS had severe symptoms, mean $\mathrm{VAS}=5.1 \pm 2.9$, compared with mean $\mathrm{VAS}=4.7 \pm 1.3$ for those without DLSS, $\mathrm{p}=0.068$.

\section{Chronicity}

Longevity of symptoms was compared between those with DLSS and those without. The average duration of symptoms for those with DLSS was $20.8 \pm 21.8$ months and for those without $12.3 \pm 15.9$ months, $p=<0.0001$. Individuals with DLSS tended to have symptoms that run a chronic cause (Table 1).

Table 1. Comparison of duration of symptoms between nonDLSS and DLSS.

\begin{tabular}{lcc}
\hline Duration (months) & Non-DLSS & DLSS \\
\hline Asymptomatic & 65 & 14 \\
$0-12$ & 152 & 4 \\
$13-24$ & 65 & 5 \\
$25-36$ & 36 & 8 \\
$37-48$ & 19 & 16 \\
$>48$ & 15 & 37 \\
Total & 352 & 84 \\
Mean duration & 13.1 & 27.6 \\
\hline
\end{tabular}

\section{DLSS and disability}

The ODI, a validated tool for health-related quality of life in patients with lower back pain, was used to measure the effects of DLSS on activities of daily living and compared with those without DLSS. The mean score in those with DLSS was $29.3 \%$ and in those without $24.8 \%$. The difference was statistically significant $(p=0.031)$. This means that those symptomatic individuals with DLSS have increased disability than patients with other forms of back pain (Table 2).

\section{Discussion}

The overall prevalence of DLSS in the study population was $19 \%$; when the symptomatic group was isolated, prevalence rose to $35 \%$. The prevalence is much higher than reported in other studies. Schroeder et al. reported a prevalence of developmental lumbar stenosis of $9.3 \%$ in the American population (14). Kalichman et al. in the Framingham study concluded that the prevalence of "congenital stenosis" was $7.3 \%$ (15). Both authors studied populations in the developed world. 
Table 2. Comparison of severity of disability using ODI score in individuals with DLSS and those without

\begin{tabular}{lcc}
\hline ODI scores (\%) & No DLSS $(n=352)$ & DLSS $(n=84)$ \\
\hline 0 (Asymptomatic) & 70 & 13 \\
$0-10$ & 13 & 4 \\
$11-20$ & 48 & 12 \\
$21-30$ & 69 & 12 \\
$31-40$ & 78 & 19 \\
$41-50$ & 54 & 18 \\
$>50$ & 20 & 7 \\
Mean & 24.8 & 29.3 \\
\hline
\end{tabular}

The cut-off in this study for radiological diagnosis of DLSS was $-2 \mathrm{SD}$ of mean parameter measurement, yielding the following values: $\mathrm{APD}<10 \mathrm{~mm}, \mathrm{TRD}<12.6 \mathrm{~mm}$ and $\mathrm{CSA}<90 \mathrm{~mm}^{2}$. For diagnosis of DLSS, Ullrich et al. in a US-based study suggested a CSA value of $<145 \mathrm{~mm}^{2}$ as a measure of 'developmental stenosis' at L3 (16) and Griffith et al. recommended a midvertebral spinal canal CSA of $<212 \mathrm{~mm}^{2}$ in males and $<213$ $\mathrm{mm}^{2}$ in females (17). A systematic review done in 2011 recommended APD of $<10 \mathrm{~mm}$ or CSA of $<90 \mathrm{~mm}^{2}$ as cutoff values for diagnosis of lumbar spinal canal stenosis (18). A study in 2012 found no standard quantitative criteria for defining anatomic lumbar spinal canal stenosis on imaging (19). However, Eisenstein et al., comparing spinal canals of Caucasians and Africans in South Africa, came up with two definitions that describe stenosis: absolute stenosis when the canal depth is less than $10 \mathrm{~mm}$ and relative stenosis when the canal depth is between $10 \mathrm{~mm}$ and $13 \mathrm{~mm}$ (20).

The average canal dimensional means found in this study were depth (APD) of $14 \mathrm{~mm}$, width (TRD) of $18 \mathrm{~mm}$, and CSA of $200 \mathrm{~mm}^{2}$. Using the global averages for comparison, the means in this study are $78 \%$ for depth, $72 \%$ for width and $57 \%$ for CSA of the Caucasian population values. Alvarez et al. studying a Caucasian population reported average lumbar spinal canal depth to be $16-20 \mathrm{~mm}$ with a mean of $18 \mathrm{~mm}$, and width of $22-28$ (mean $=25 \mathrm{~mm}$ ), yielding a cross-sectional area of $276-440 \mathrm{~mm}^{2}$ (mean=350 $\mathrm{mm}^{2}$ ) (21). Griffith et al. reporting a study from a Korean population found a mean APD of $15.8 \pm 1.96 \mathrm{~mm}$, canal width of $23.6 \pm 4.25 \mathrm{~mm}$ and CSA of $278.5 \pm 50.3 \mathrm{~mm}^{3}(17)$. There are few published values from the indigenous African population for comparison.

This study shows that individuals with DLSS have a higher frequency of LBP (82\%) than those without DLSS (66\%). Similarly, individuals with DLSS have a higher frequency of radiculopathy (90\%) than those without (48\%). The prevalence of these symptoms is high even in the study population, $72 \%$ and $56 \%$ for LBP and radiculopathy respectively, and can be explained by the sample bias of preselecting individuals with or seeking lumbosacral scanning.

A significant correlation is observed between DLSS, chronicity, disability and level of pain. The severity of these symptoms was compared in those with DLSS against those without. All outcome measures - pain intensity, chronicity, and disability showed significant differences in those with DLSS, revealing prolonged, painful and disabling symptoms. Zucherman et al. in a prospective randomized multi-center study for the treatment of lumbar spinal stenosis with the X-STOP interspinous implant used the same outcome measures (Zurich Claudication Questionnaire) to monitor the outcome of treatment of spinal stenosis (22). Other studies have used patient-reported outcome measures such as ODI and VAS (23). The baseline duration of symptoms for those with DLSS in this study was $20.8 \pm 21.8$ months and $12.3 \pm 15.9$ months for those without $(p<0.0001)$. The level of disability was worse in those with DLSS, mean ODI $32.9 \pm 15.2$, than in those without, mean ODI $17.2 \pm 16.5$ $(\mathrm{p}<0.0001)$. The visual analogue scale showed those with DLSS had more severe symptoms (VAS $=5.1 \pm 2.9$ ) than those without DLSS, VAS $=4.7 \pm 1.3, \mathrm{p}=0.068$.

\section{Conclusion}

Individuals with radiological DLSS are more symptomatic than those without. These individuals are disadvantaged because they have a pre-existing narrow canal which does not exempt them from degenerative changes and other space-occupying lesions. When these conditions occur, compression is more severe than in the individuals with large canals. Radiological diagnosis of DLSS is therefore paramount as these individuals will need a different approach in management.

\section{Acknowledgements}

I thank my supervisors for their guidance during the course of this study. I also thank the hospitals that allowed me to collect data in their radiology departments, and the study assistants who tirelessly helped to collect the data.

\section{References}

1. Arnoldi C, Brodsky A, Cauchoix J, Crock H, et al. Lumbar spinal stenosis and nerve root entrapment syndromes. Definition and classification. Clin Orthop Relat Res. 1976; 115:4-5.

2. Lasanianos N, Triantafyllopoulos G, Pneumaticos S. Lumbar stenosis. In: Lasanianos N, Kanakaris N, Giannoudis P, editors. Trauma and orthopaedic classifications. London: Springer. 2015; 247-50.

3. Verbiest H. A radicular syndrome from developmental narrowing of the lumbar vertebral canal. J Bone Joint Surg Br. 1954; 36:230-7.

4. Clark G, Panjabi M, Wetzel F. Can infant malnutrition cause adult vertebral stenosis? Spine. 1985; 10:165-70.

5. Jeffrey J, Campbell D, Golden M, et al. Antenatal factors in the development of the lumbar vertebral canal: A magnetic resonance imaging study. Spine. 2003; 28:1418-23.

6. Watts R. Lumbar vertebral canal size in adults and children: Observations from a skeletal sample from London, England. HOMO-J Comp Hum Biol. 2013; 64:120-8. 
7. Papp T, Porter R, Aspden R. The growth of the lumbar vertebral canal. Spine. 1994; 19:2770-3.

8. Epstein J, Epstein B, Lavine L. Nerve root compression associated with narrowing of the lumbar spinal canal. J. Neurol. Neurosurg. Psychiatry. 1962; 25:165-76.

9. Ehni G. Significance of the small lumbar spinal canal: Cauda equina compression syndromes due to spondylosis. J Neurosurg. 1969; 31:490-4.

10. Porter R, Ward D. Cauda equina dysfunction: The significance of two-level pathology. Spine. 1992; 17:9-15.

11. Kreiner D, Scott S, William O, et al. An evidence-based clinical guideline for the diagnosis and treatment of degenerative lumbar spinal stenosis (update). Spine J. 2013; 13:734-43.

12. Modic M, Masaryk T, Ross J, et al. Imaging of degenerative disk disease. Radiology. 1988; 168:177-86.

13. Schizas C, Schmit A, Schizas A, et al. Secular changes of spinal canal dimensions in Western Switzerland: A narrowing epidemic? Spine. 2014; 39:1339-44.

14. Schroeder G, Kurd M, Vaccaro A. Lumbar spinal stenosis: How is it classified? J Am Acad Orthop Surg. 2016; 24:843-52.

15. Kalichman L, Cole R, David H, et al. Spinal stenosis prevalence and association with symptoms: The Framingham study. Spine J. 2009; 9:545-50.

16. Ullrich G, Binet E, Sanecki M, et al.: Quantitative assessment of the lumbar spinal canal by computed tomography. Radiology. $1980 ; 134: 137-43$.
17. Griffith J, Huang J, Sheung W, et al. Population reference range for developmental lumbar spinal canal size. Quant Imaging Med Surg. 2016; 6:671-9.

18. Steurer J, Simon G, Ralph H, et al. Quantitative radiologic criteria for the diagnosis of lumbar spinal stenosis: A systematic literature review. BMC Musculoskelt Disord. 2011; 12:175.

19. Mamisch N, Brumann M, Hodler J, et al. Radiologic criteria for the diagnosis of spinal stenosis: results of a Delphi survey. Radiology. 2012; 264:174-9.

20. Eisenstein S. The morphometry and pathological anatomy of the lumbar spine in South African negroes and caucasoids with specific reference to spinal stenosis. J Bone Joint Surg Br. 1977; 59:173-80.

21. Alvarez J, Hardy Jr, Russell H. Lumbar spine stenosis: A common cause of back and leg pain. Am Fam Physician. 1998; 57:1825-34.

22. Zucherman J, Hsu K, Hartjen C, et al. A prospective randomized multi-centre study for the treatment of lumbar spinal stenosis with the X STOP interspinous implant: 1-year results. Eur Spine J. 2004; 13:22-31.

23. Fritsch C, Ferreira M, Maher C, et al. The clinical course of pain and disability following surgery for spinal stenosis: A systematic review and meta-analysis of cohort studies. Eur Spine J. 2017; 26:324-35.

24. Katindi S. Exploring Kenya's: Inequality pulling apart or pooling together? A publication of KNBS and SID. 2013. ISBN 9966029184 